\title{
Functional follow-up after endoscopic calcaneoplasty for Haglund's deformity using the biodex isokinetic muscle testing system: A case series
}

\author{
YU-PING YANG $^{1 *}$, BING-HUA LI $^{1,2^{*}}$, YI-XIAO WEI $^{1,3}$, XIAO-YU PAN $^{1,4}$, LI-YUAN TAO ${ }^{5}$, \\ NING AN ${ }^{6}$, YAN-BING JIA ${ }^{1,7}$, LI-LI LI ${ }^{1,8}$ and YING-FANG AO ${ }^{1}$ \\ ${ }^{1}$ Institute of Sports Medicine, Peking University Third Hospital, Beijing 100191; ${ }^{2}$ Shenzhen Graduate School, \\ Peking University, Shenzhen, Guangdong 518055; ${ }^{3}$ Peking University Health Science Center, Peking University; \\ ${ }^{4}$ Department of Orthopedics and ${ }^{5}$ Research Center of Clinical Epidemiology, Peking University Third Hospital; Schools of \\ ${ }^{6}$ Pharmaceutical Science, ${ }^{7}$ Public Health and ${ }^{8}$ Basic Medical Sciences, Peking University, Beijing 100191, P.R. China
}

Received January 20, 2020; Accepted June 3, 2020

DOI: $10.3892 /$ etm.2020.9006

\begin{abstract}
Isokinetic muscle strength test implemented by the Biodex system is a method used for evaluating muscle function that has been applied clinically in the field of sports and rehabilitation medicine. However, information on its application on Haglund's deformity remain insufficient. Therefore, the present study examined the effectiveness of the muscle strength test using the Biodex system in evaluating the recovery of athletic capacity in patients with Haglund's deformity following endoscopic surgery. In total, 34 patients treated by the authors from June 2012 to November 2018 at Peking University Third Hospital (Beijing, China) were included. To compare muscle strength before surgery, then 3 and 6 months after surgery, using the uninjured side as the control, the Biodex system test was conducted in parallel to the collection of the American Orthopaedic Foot and Ankle Score values and visual analog scale scores. The Biodex system test results showed that Haglund's deformity mainly hinders plantar flexion strength. Patients recovered daily living capacity within 3 months and athletic capacity within 6 months following surgery, which matched the AOFAS values, VAS scores and the self-assessments of the patients. These findings suggest that the Biodex system can dynamically reflect the degree of postoperative recovery in Haglund's deformity.
\end{abstract}

Correspondence to: Professor Ying-Fang Ao, Institute of Sports Medicine, Peking University Third Hospital, 49 Huayuan Road, Beijing 100191, P.R. China

E-mail: aoyingfang@yeah.net

${ }^{*}$ Contributed equally

Key words: Haglund's deformity, isokinetic muscle testing, endoscopy, biodex system, calcaneoplasty

\section{Introduction}

Posterior ankle pain is a multifaceted condition that can pose a major concern among young and middle-aged patients (1). Haglund's deformity, first described by the Swedish physician Patrick Haglund in 1928 (2), is defined as an inflammation of the bursa in the posterior calcaneus, which can lead to repetitive mechanical impacts between the posterior calcaneus and Achilles tendon (3). The inflammation can cause pain and swelling, which may affect the patient's ability to work or exercise (4).

Both conservative and surgical methods are used for the treatment of Haglund's deformity. Patients showing poor response to conservative treatment can undergo surgery to remove the abnormally prominent posterior calcaneus and the subcondylar inflammatory bursa (5). Due to its continual development and improvements, endoscopy is becoming increasingly popular $(6,7)$, which can be performed using two- and three-portal techniques (8).

Isokinetic muscle strength test implemented on the Biodex system (9) is a method used for evaluating and testing muscle function that has been applied in clinical research in sports and rehabilitation medicine $(10,11)$. However, to the best of our knowledge, it has not been previously applied for evaluating Haglund's deformity. This test can be applied to the plantar flexors and dorsiflexors of the ankle joint to evaluate muscle strength and endurance, providing reliable clinical evidence of patient rehabilitation after endoscopic calcaneoplasty (12). In the present study, Biodex system test results, American Orthopaedic Foot and Ankle Score (AOFAS) values and visual analog scale (VAS)scores $(13,14)$ were all obtained from each patient to compare muscle strength and performance before surgery, then 3 and 6 months after surgery. The aim of the present study was to assess the effectiveness of the Biodex system in determining postoperative recovery for treating Haglund's deformity.

\section{Patients and methods}

Patients. A retrospective (medical records), level 3 evidence study was performed to evaluate the outcomes of patients who 
underwent endoscopic surgery for Haglund's deformity. The present study included 34 patients (age range, 15-44 years; mean age, $31.3 \pm 11.6$ years; sex, 30 men and 4 women) who were diagnosed with Haglund's deformity and were treated by the authors from June 2012 to November 2018 at the Peking University Third Hospital (Beijing, China). All patients, 2 of whom were professional athletes, underwent conservative therapy initially, including physical therapy, calf and ankle muscle strength exercises and oral anti-inflammatory analgesics. Patients aged $>45$ years, suffering with diseases involving the Achilles tendon and with Achilles tendon rupture were all excluded. The present study was approved by the Peking University Third Hospital's ethics committee. Written informed consent was obtained from all patients, parents or guardians of the patients prior to the present study. The rights of the patients were protected.

Diagnosis. Patients were diagnosed based on subjective complaints, combined with clinical and radiological examinations. Radiological measurements for this disease include the Fowler-Phillip angle, calcaneal pith angle, parallel pitch lines, Chauveaux-Liet angle and the X/Y ratio (15-19). Haglund's deformity would be suspected in cases where the lateral angle between the plantar and posterior borders of the calcaneus, also known as Fowler-Phillip angle, $>75^{\circ}(15-19)$. For the examinations performed in the present study, the upper part of the calcaneal nodule (noncalcaneus nodules) exhibited obvious tenderness at $2-3 \mathrm{~cm}$ and posterior protrusion of the calcaneus. MRI confirmed the abnormal ridge of the posterior calcaneus and the presence of collateral fluid in the Achilles tendon. Suspected peep or partial tearing of the Achilles tendon was observed in some patients. All patients exhibited soft tissue swelling in or around the Achilles tendon and pain in the stretched tendon.

Surgical technique. To prepare for the endoscopic procedure, each patient was placed in the prone position under lumbar anesthesia. In the present study, endoscopic calcaneoplasty was performed using the two-portal technique. A tourniquet was applied to the lower extremity, where 5-mm-long portals were made on the inner and outer edges of the Achilles tendon at the posterior calcaneus level to insert the endoscopes and surgical instruments. Surgical instruments applied in the present study were as follows: i) Endoscope, Smith \& Nephew 560P High Definition Camera System; ii) power equipment for blades and burr, Smith \& Nephew Dyonics Power II Control System; iii) blades, Smith \& Nephew Dyonics $4.5 \mathrm{~mm}$ Synoblator blade platinum series (cat. no. 72203523); and iv) burr (cat. no. 7205324), Smith \& Nephew Dyonics $4.0 \mathrm{~mm}$ abrader burr (All from Smith \& Nephew plc.). In general, endoscopes were inserted directly into the anterior sac of the Achilles tendon to observe the inflammation of the synovium. For some patients in the present study, planers were required to clean portions of the adipose tissue on the ventral side so that the Achilles tendon and calcaneus could be revealed completely. The cartilage of the posterior superior calcaneus from $64.3 \%$ of the patients was observed to be degenerated (Fig. 1). During ankle dorsiflexion, posterior calcaneus collided with the Achilles tendon in all patients (Fig. 2). The prominent posterior calcaneus was then polished to a flat

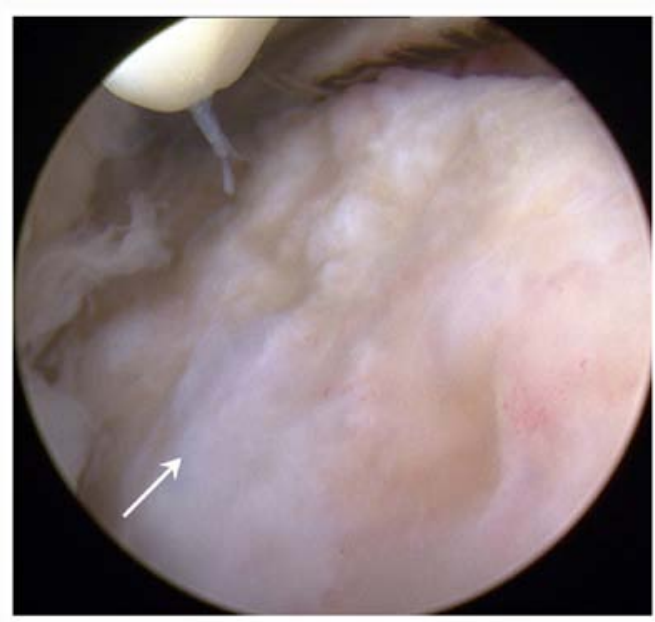

Figure 1. Arthroscopic view of the degenerated cartilage of the posterior superior calcaneus. Arrow indicates the damaged cartilage-like surface of the exostosis

or slightly concave shape using a burr and the distal end of the calcaneus in front of the Achilles tendon was resected (Figs. 3 and 4). This can completely halt the collision action on dorsiflexion. Finally, the portals were flushed and closed using pressure-wrapped cotton and a splint.

Postoperative care. The plaster was removed 3 weeks after surgery. The patients were instructed to wore shoes with a 2-cm-thick pad to walk using their full weight for 9 weeks, whilst scheduled activities such as lifting exercises were performed in parallel. Recreational activities or special training was gradually resumed after 3 months.

Anthropometric measurements. Isokinetic strength tests were conducted using the Biodex system 1 week before surgery and then 3 and 6 months after surgery. Accordingly, AOFAS values and VAS scores were also obtained preoperatively and at 3- and 6-month follow-ups. MRI and X-ray were used to examine the patients after 5 months. Self-evaluations of recovery were also recorded from each patient, including the time taken for the recovery of daily life (months), recovery of abilities to exercise (months) and work (months).

All isokinetic strength tests were performed using the same dynamometer (Biodex System 3-Dynamometer; Biodex Medical Systems, Inc). The dynamometer was used to measure the maximum torque of the affected ankle at 60 and $120 \% \mathrm{sec}$ before surgery and 3 and 6 months after surgery. The patients performed a low-resistance warm-up for 5 min on a bicycle before the test. Seated in a chair at $90^{\circ}$, each patient's lower limbs were weighed before the test, following which the results were adjusted in accordance with the weight of the lower limbs to exclude the effect of gravity during limb movement. The total range of motion was $120^{\circ}$, set from $120^{\circ}$ to $0^{\circ}$ of leg flexion. Each patient performed three submaximal contractions at $60 \%$ sec for warm-up, followed by a 30 -sec rest before five maximal contractions at $60 \% \mathrm{sec}$. After a 1 -min rest, the patients then performed three submaximal contractions at $180 \% \mathrm{sec}$, followed by a 30 -sec rest before five maximal contractions at $180 \% \mathrm{sec}$. The uninjured side was tested first, 


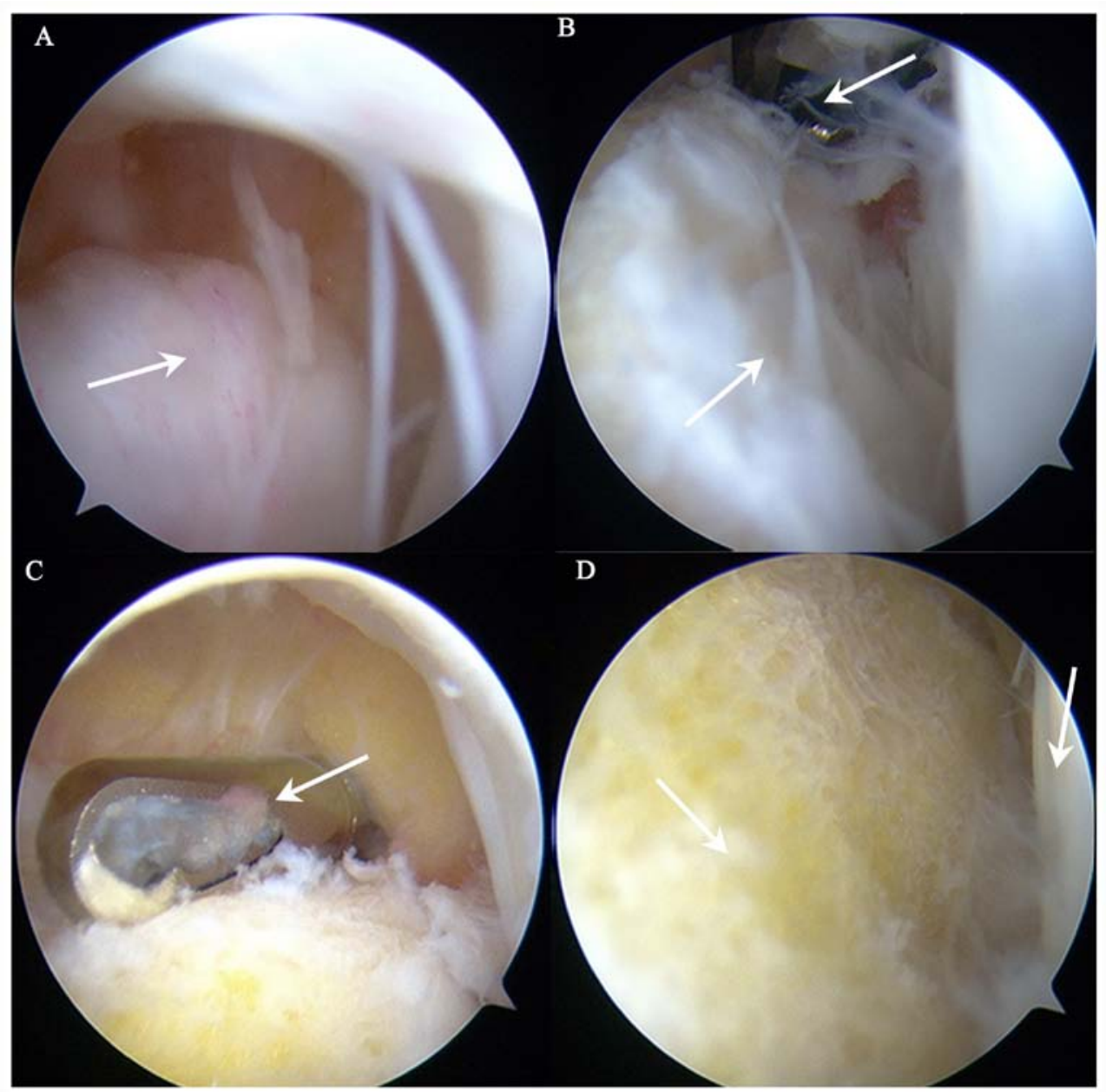

Figure 2. Representative images showing the intraoperative and postoperative arthroscopic view. (A) Significant effusion as revealed by an intraoperative arthroscopic exploration of the Achilles tendon sac. Arrow indicates the synovium on the surface of the calcaneum. (B) Intraoperative angioplasty revealing nodular swelling of the posterior calcaneus. Upper arrow indicates the location of the burr used during endoscopic calcaneoplasty for Haglund deformity. Lower arrow indicates the preoperative surface of the posterior calcaneus with nodules. (C) Nodules formed on the posterior calcaneus. Arrow indicates location of the cutter cleaning up bone debris after calcaneoplasty. (D) Nodules formed on the posterior calcaneus. The posterior calcaneus and the Achilles tendon no longer collided. Left arrow indicates the postoperative posterior calcaneus with nodules. Right arrow indicates ventral side of the insertion of the Achilles tendon.

the results of which were used as the control. The injured side was tested within 2 min after testing the uninjured side.

Data analysis. The peak extension and flexion torques for both ankles as obtained using the Biodex system, along with AOFAS values and VAS scores, were evaluated for all patients. Wilcoxon signed rank test was used to analyze the difference between the uninjured and injured sides in terms of the AOFAS values and VAS scores evaluated preoperatively and those evaluated at the 3 and 6 months postoperatively. Friedman's test followed by Nemenyi's test with Bonferroni's correction was performed for comparing the AOFAS and VAS scores before the operation, 3 and 6 months after the operation. $\mathrm{P}<0.05$ was considered to indicate a statistically significant difference. Statistical analysis was performed using the SPSS software (version 23.0; IBM Corp.).

\section{Results}

Biodex system results. Isokinetic strength test conducted on both the injured and uninjured sides of the patients using the Biodex system before surgery, then 3 and 6 months after surgery. No significant differences were observed between the injured and uninjured sides in dorsiflexion torque at 60 or $120 \%$ sec (Tables I and II). Conversely, significant differences were observed between the injured and uninjured sides in plantarflexion torque at 120 and $60 \%$ sec before surgery $(\mathrm{P}=0.036$ and $\mathrm{P}=0.040$, respectively). At 3 months after surgery, a significant difference remained between the two sides tested in plantarflexion torque at $120 \% \mathrm{sec}(\mathrm{P}=0.025)$. However, the difference between the two sides at $60 \% \mathrm{sec}$ was not found to be significant $(\mathrm{P}=0.459)$. At 6 months after surgery, no significant differences were found between the injured and uninjured sides in plantarflexion torque at 120 or $60^{\circ} / \mathrm{sec}$.

AOFAS and VAS results. The AOFAS values and VAS results obtained at the follow-up time points exhibited a consistent trend with the results obtained from the Biodex system (Table III). Significant differences were observed among in those before surgery, 3 and 6 months after surgery $(\mathrm{P}<0.001$ and $\mathrm{P}<0.001$, respectively). Specifically, compared with those before surgery, the AOFAS values and VAS scores increased, 

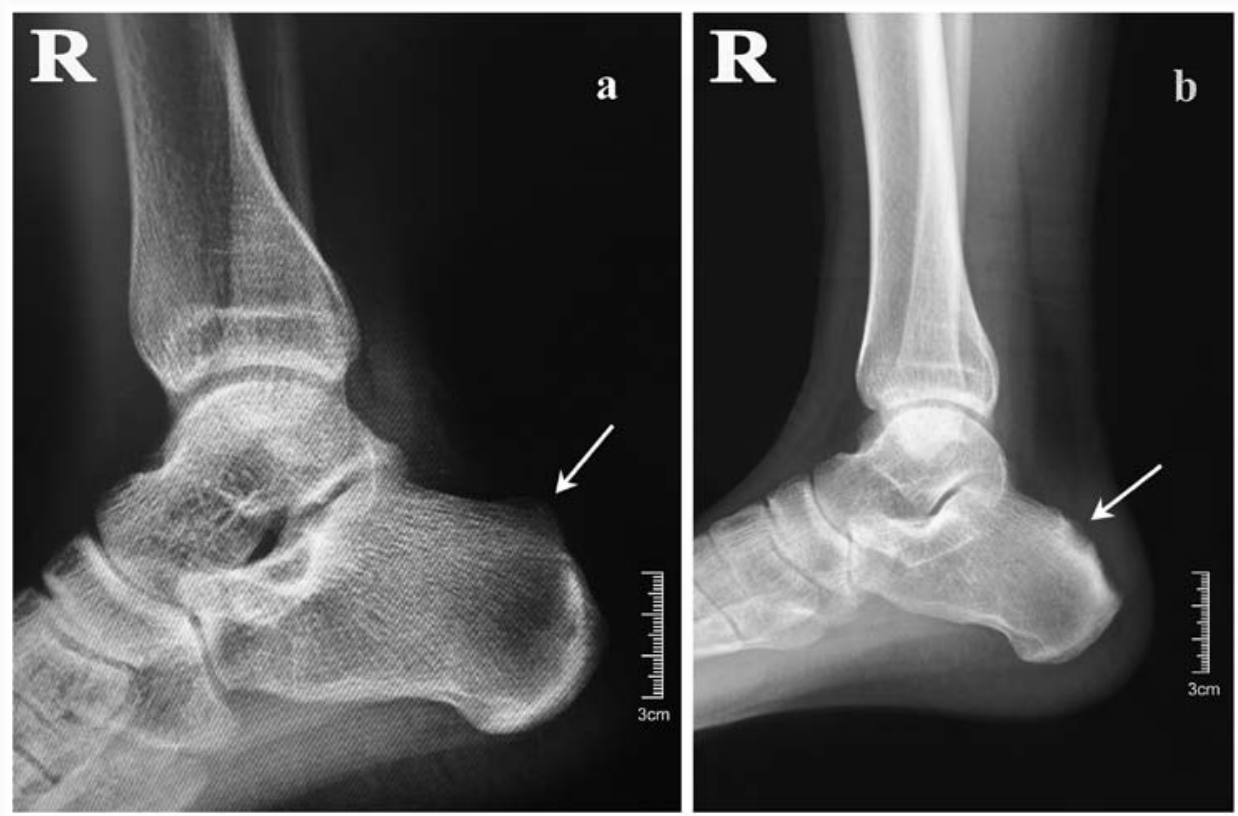

Figure 3. Representative preoperative and postoperative lateral radiographs. (A) Lateral radiograph taken 1 month prior to operation showing substantial posterosuperior calcaneal prominence. Arrow indicates location of the nodule. (B) Lateral radiograph taken 5 months after operation showing the complete resection of the bony prominence. Arrow indicates the location of the deformed surface. $\mathrm{R}$, right foot.
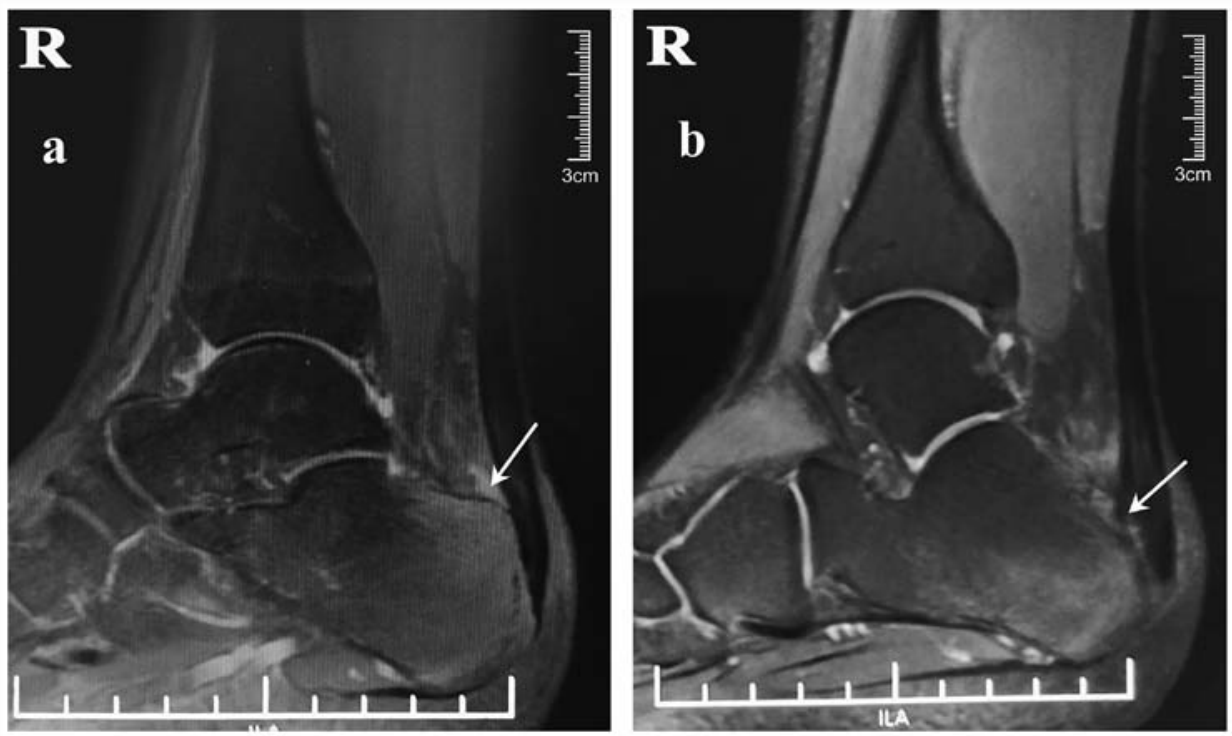

Figure 4. Preoperative and postoperative sagittal plane MRI images. (A) Sagittal plane MRI taken 1 month prior to operation showing notable posterosuperior calcaneal prominence. Arrow indicates the swollen synovium on the ventral side of the Achilles tendon caused by effusion due to inflammation. (B) Sagittal plane MRI taken 5 month prior to operation showing complete resection of the bony prominence. Arrow indicates the location of the postoperative posterior calcaneus with the prominent parts removed, where the swollen inflamed synovium also disappeared.

3 and 6 months after surgery. At 3 months, the AOFAS values and VAS results reached 89.0 $\pm 3.8 / 100$ (range, 79-95; $\mathrm{P}<0.001$ vs. before surgery) and $3.1 \pm 0.6 / 10$ (range, 2-5; $\mathrm{P}=0.002$ vs. before surgery), respectively. At 6 months, the AOFAS values and VAS scores reached 93.7 $\pm 3.3 / 100$ (range, $85-100 ; \mathrm{P}<0.001$ vs. before surgery; $\mathrm{P}<0.001$ vs. 3 months after surgery) and 1.5 $\pm 0.4 / 10$ (range, $1-2 ; \mathrm{P}<0.001$ vs. before surgery; $\mathrm{P}<0.001$ vs. 3 months after surgery), respectively. This corresponded to an improvement of 29.3 in the AOFAS value and a reduction of 3.0 points in the VAS from the mean preoperative scores.
Patient self-assessment. The results of the patient self-assessment were also found to be consistent with the results obtained using the Biodex system. During the follow-up period, the patients reported that they recovered living and athletic capacity within $2.7 \pm 1.5$ and $6.6 \pm 3.0$ months, respectively, on average. MRI was used to confirm the abnormal ridge of the posterior calcaneus and the collateral fluid in the Achilles tendon during the follow-up period (Fig. 4). Most patients reported alleviation of pain at the final follow-up. Specifically, in terms of pain 15 patients recovered within 3 months, 17 recovered within 4-6 months, but 2 showed no recovery. 
Table I. Comparison of torque at $120^{\circ} / \mathrm{sec}$ between the injured and uninjured sides of 34 patients before and 3 and 6 months after surgery.

\begin{tabular}{|c|c|c|c|c|c|c|}
\hline \multirow[b]{2}{*}{ Time } & \multicolumn{2}{|c|}{ Plantar flexor } & \multirow[b]{2}{*}{ P-value } & \multicolumn{2}{|c|}{ Dorsal flexor } & \multirow[b]{2}{*}{ P-value } \\
\hline & Uninjured & Injured & & Uninjured & Injured & \\
\hline Before surgery & $28.5 \pm 11.6$ & $22.3 \pm 7.2$ & 0.036 & $17.9 \pm 10.1$ & $14.5 \pm 6.4$ & 0.073 \\
\hline 3 months after surgery & $29.5 \pm 15.2$ & $22.9 \pm 10.1$ & 0.025 & $18.2 \pm 10.3$ & $15.4 \pm 9.1$ & 0.099 \\
\hline 6 months after surgery & $30.4 \pm 16.1$ & $25.7 \pm 11.4$ & 0.061 & $17.4 \pm 6.2$ & $16.0 \pm 8.4$ & 0.151 \\
\hline
\end{tabular}

Table II. Comparison of torque at $60^{\circ} / \mathrm{sec}$ between the injured and uninjured sides of 34 patients before and 3 and 6 months after surgery.

\begin{tabular}{|c|c|c|c|c|c|c|}
\hline \multirow[b]{2}{*}{ Time } & \multicolumn{2}{|c|}{ Plantar flexor } & \multirow[b]{2}{*}{ P-value } & \multicolumn{2}{|c|}{ Dorsal flexor } & \multirow[b]{2}{*}{ P-value } \\
\hline & Uninjured & Injured & & Uninjured & Injured & \\
\hline Before surgery & $26.5 \pm 9.6$ & $20.8 \pm 6.0$ & 0.040 & $19.8 \pm 12.0$ & $16.5 \pm 8.9$ & 0.579 \\
\hline 3 months after surgery & $27.2 \pm 11.2$ & $25.6 \pm 15.1$ & 0.459 & $18.7 \pm 12.2$ & $7 \pm 9.6$ & 0.786 \\
\hline 6 months after surgery & $27.7 \pm 10.6$ & $26.5 \pm 16.3$ & 0.827 & $20.9 \pm 9.7$ & $19.4 \pm 10.7$ & 0.290 \\
\hline
\end{tabular}

Table III. AOFAS values and VAS scores obtained from the 34 patients 1 week before and 3 and 6 months after surgery.

\begin{tabular}{lrrr}
\hline Score type & Pre-operation & $\begin{array}{c}3 \text { months } \\
\text { after surgery }\end{array}$ & $\begin{array}{r}6 \text { months } \\
\text { after surgery }\end{array}$ \\
\hline AOFAS & $64.4 \pm 4.5$ & $89.0 \pm 3.8^{\mathrm{a}}$ & $93.7 \pm 3.3^{\mathrm{c}, \mathrm{e}}$ \\
VAS & $4.5 \pm 0.9$ & $3.1 \pm 0.6^{\mathrm{b}}$ & $1.5 \pm 0.4^{\mathrm{d}, \mathrm{f}}$ \\
\hline
\end{tabular}

${ }^{\mathrm{a}} \mathrm{P}<0.001 \mathrm{vs}$. before surgery. ${ }^{\mathrm{b}} \mathrm{P}=0.002 \mathrm{vs}$. before surgery. ${ }^{\mathrm{c}} \mathrm{P}<0.001 \mathrm{vs}$. before surgery. ${ }^{\mathrm{d}} \mathrm{P}<0.001$ vs. before surgery. ${ }^{\mathrm{e}} \mathrm{P}<0.001$ vs. 3 months after surgery. ${ }^{\mathrm{f}} \mathrm{P}<0.001$ vs. 3 months after surgery. AOFAS, American Orthopaedic Foot and Ankle Score; VAS, visual analog scale.

\section{Discussion}

A number of studies have previously reported isokinetic dynamometry to be a reliable procedure for evaluating ankle joint function (20-22). Although studies have been performed using isokinetic dynamometry to evaluate ankle function in patients with other diseases, including sciatica (23), and rheumatoid arthritis (24), studies applying this procedure to assess Haglund's deformity remain insufficient. In general, torque testing at 60 and $120 \% \mathrm{sec}$ are associated with strength and power parameters, respectively (25). In the present study conducted on patients with Haglund's deformity, the results revealed no significant differences between the injured and uninjured sides in terms of dorsiflexion torque at 60 or $120 \% \mathrm{sec}$. these findings indicate that this disease does not hinder dorsiflexion strength but instead affects plantarflexion strength. Before surgery, the plantarflexion torque at 60 and $120 \%$ sec varied significantly between the injured and uninjured sides. At 3 months after surgery, the plantarflexion torques at $60 \%$ sec were almost identical on both sides, suggesting no significant difference. However, torques at $120 \%$ sec differed significantly between the two sides 3 months after surgery. This indicates that although patients had recovered extensor muscle strength for slower movements, the ability to adapt to fast movements was not yet recovered within 3 months of surgery. Although their normal day-to-day functions were restored, difficulties remained in resuming work. The results showed no statistically significant differences in plantarflexion torque at 60 or $120 \%$ sec between the two sides 6 months after surgery. The strength of the extensors on the injured side was found to be almost identical compared with that on the uninjured side, such that the patients could resume normal exercise and work. The AOFAS values and VAS scores in addition to patient self-assessment regarding recovery time also exhibited a consistent trend with the Biodex results during the follow-up period, further demonstrating the validity of the results obtained using the Biodex system.

Conventional treatment for Haglund's deformity involves resting, wearing modified shoes, medical therapy and in selected cases, surgery (26). Open surgery is no longer executed due to the trauma it causes and the relatively high risk of infection (27). Endoscopic surgery is commonly performed and has been reported to engender notable improvements with limited complication rates of ankle surgeries (28-30). Additionally, it also provides an optimal view of the pathological posterosuperior calcaneal region using small incisions.

Results from the present study also confirmed that the postoperative care plan was reasonable and effective. The patients could wear shoes with 2-cm-thick pads 3 weeks after surgery for 9 weeks, perform some exercises and walk using their entire weight. After 3 months, the patients reported recovery of living capacity, where they gradually resumed recreational activities or special training. Recovery of athletic capacity was reported after 6 months. 
Limitations exist regarding the present study. The sample size was relatively small and therefore more cases are required to obtain more representative research findings. The diagnosis of Haglund's deformity of the Achilles tendon remains controversial. Recognized diagnostic criteria have yet to be established, where physicians perform diagnoses subjectively on the basis of their own judgment. Although the Fowler-Philip angle was measured as a diagnostic factor, previous studies have revealed that its correlation with clinical symptoms is low, with the false negative rate reaching $85-100 \%$. As a result, its accuracy as a diagnostic and predictive indicator remains poor (31). Additionally, the Fowler-Philip angle exceeded $75^{\circ}$ in only three patients in the present study, further suggesting its unreliability in diagnosing Haglund's deformity.

Pavlov et al (16) considered that the degree of bulging of the posterior calcaneus is the most important symptom when the parallel pitch line is used as a measurement index. The degree of kyphosis of the calcaneus exceeded the parallel pitch line in all patients in the present study, suggesting that the parallel pitch line is more reliable for diagnosing Haglund's deformity. MRI can also clearly show the degree of kyphosis, its relationship with the Achilles tendon and the swelling of the Achilles tendon. Therefore, Haglund's deformity was diagnosed comprehensively in the present study by evaluating the clinical manifestations, X-ray examination and MRI results.

To conclude, findings from the present study suggest that using the Biodex system for isokinetic muscle testing can dynamically reflect the postoperative recovery of patients with Haglund's deformity after endoscopic calcaneoplasty.

\section{Acknowledgements}

The authors would like to thank Dr Zhai Wenbo (School of Basic Medical Sciences, Peking University; Beijing, China) for his immense effort and guidance in the statistical analyses of this study.

\section{Funding}

No funding was received.

\section{Availability of data and materials}

The datasets used and/or analyzed during the current study are available from the corresponding author on reasonable request.

\section{Authors' contributions}

YPY and YFA performed the surgical procedures. YXW, YFA, YPY and BHL designed the study. BHL, NA, YBJ and LLL contributed to patient data collection and follow-up. BHL, LYT and XYP analyzed the data and performed the statistical analysis. YXW is accountable for all aspects of the work. All authors read and approved the final manuscript.

\section{Ethics approval and consent to participate}

The present study was approved by the Peking University Third Hospital Medical Science Research Ethics Committee (approval no. IRB00006761-M2018151; Beijing, China). Written informed consent was obtained from all patients, parents or guardians of the patients prior to the present study.

\section{Patient consent for publication}

Not applicable.

\section{Competing interests}

The authors declare that they have no competing interests.

\section{References}

1. Ögüt T and Yontar NS: Treatment of hindfoot and ankle pathologies with posterior arthroscopic techniques. EFORT Open Rev 2: 230-240, 2017.

2. Haglund P: Beitrag zur klinik der achillessehne. Zeitschr Orthop Chir 49: 49-58, 1928.

3. Wiegerinck JI, Kok AC and van Dijk CN: Surgical treatment of chronic retrocalcaneal bursitis. Arthroscopy 28: 283-293, 2012.

4. Ortmann FW and McBryde AM: Endoscopic bony and soft-tissue decompression of the retrocalcaneal space for the treatment of Haglund deformity and retrocalcaneal bursitis. Foot Ankle Int 28: 149-153, 2007.

5. Mochizuki Y, Saito Y, Tsujikawa T, Fujiyama Y and Andoh A: Combination of endoscopic submucosal dissection and chemoradiation therapy for superficial esophageal squamous cell carcinoma with submucosal invasion. Exp Ther Med 2: 1065-1068, 2011.

6. Zhou XN, Li B, Wang JS and Bai LH: Surgical treatment of popliteal cyst: A systematic review and meta-analysis. J Orthop Surg Res 11: 22, 2016.

7. Vega J, Baduell A, Malagelada F, Allmendinger $J$ and Dalmau-Pastor M: Endoscopic achilles tendon augmentation with suture anchors after calcaneal exostectomy in haglund syndrome. Foot Ankle Int 39: 551-559, 2018.

8. Uzümcügil O, Doğan A, Yalçinkaya M, Mumcuoğlu E and Kabukçuoğlu Y: The three portal (including transpatellar tendon portal) versus two portal technique in the arthroscopic menisectomy procedure for isolated medial bucket-handle type meniscal tears. Eklem Hastalik Cerrahisi 21: 38-43, 2010.

9. Taylor NA, Sanders RH, Howick EI and Stanley SN: Static and dynamic assessment of the biodex dynamometer. Eur J Appl Physiol Occup Physiol 62: 180-188, 1991.

10. Papandreou MG, Billis EV, Antonogiannakis EM and Papaioannou NA: Effect of cross exercise on quadriceps acceleration reaction time and subjective scores (Lysholm questionnaire) following anterior cruciate ligament reconstruction. J Orthop Surg Res 4: 2, 2009.

11. Müller M, Disch AC, Zabel N, Haas NP and Schaser KD: Initial intramuscular perfusion pressure predicts early skeletal muscle function following isolated tibial fractures. J Orthop Surg Res 3: $14,2008$.

12. Sung E and Kim J: Relationship between ankle range of motion and biodex balance system in females and males. J Exerc Rehabil 14: 133-137, 2018.

13. Kitaoka HB, Alexander IJ, Adelaar RS, Nunley JA, Myerson MS and Sanders M: Clinical rating systems for the ankle-hindfoot, midfoot, hallux, and lesser toes. Foot Ankle Int 15: 349-353, 1994.

14. Hayes MH and Patterson DG: Experimental development of the graphic rating method. Psychol Bull 18: 98-99, 1921.

15. Fowler A and Phillip J: Abnormality of the calcaneus as acause of painful heel: Its diagnosis and operative treatment. Br J Surg 132: 494-498, 1945.

16. Pavlov H, Heneghan MA, Hersh A, Goldman AB and Vigorita V: The Haglund syndrome: Initial and differential diagnosis. Radiology 144: 83-88, 1982.

17. Chauveaux D, Liet P, Le Huec JC and Midy D: A new radiologic measurement for the diagnosis of Haglund's deformity. Surg Radiol Anat 13: 39-44, 1991.

18. Tourné Y, Baray AL, Barthélémy R and Moroney P: Contribution of a new radiologic calcaneal measurement to the treatment decision tree in Haglund syndrome. Orthop Traumatol Surg Res 104: 1215-1219, 2018. 
19. Lu CC, Cheng YM, Fu YC, Tien YC, Chen SK and Huang PJ: Angle analysis of Haglund syndrome and its relationship with osseous variations and Achilles tendon calcification. Foot Ankle Int 28: 181-185, 2007.

20. Ryan ED, Beck TW, Herda TJ, Hull HR, Hartman MJ, Costa PB, Defreitas JM, Stout JR and Cramer JT: The time course of musculotendinous stiffness responses following different durations of passive stretching. J Orthop Sports Phys Ther 38: 632-639, 2008.

21. Harbo T, Brincks J and Andersen H: Maximal isokinetic and isometric muscle strength of major muscle groups related to age, body mass, height, and sex in 178 healthy subjects. Eur J Appl Physiol 112: 267-275, 2012.

22. de Araujo Ribeiro Alvares JB, Rodrigues R, de Azevedo Franke R, da Silva BG, Pinto RS, Vaz MA and Baroni BM: Inter-machine reliability of the biodex and cybex isokinetic dynamometers for knee flexor/extensor isometric, concentric and eccentric tests. Phys Ther Sport 16: 59-65, 2015.

23. Ustun N, Erol O, Ozcakar L, Ceceli E, Ciner OA and Yorgancioglu ZR: Association with isokinetic ankle strength measurements and normal clinical muscle testing in sciatica patients. J Back Musculoskelet Rehabil 26: 361-365, 2013.

24. Oliveira SC, Oliveira LM, Jones A and Natour J: Isokinetic assessment of ankles in patients with rheumatoid arthritis. Rev Bras Reumatol 55: 318-324, 2015 (In Portuguese).

25. Dervin GF, Taylor DE and Keene GC: Effects of cold and compression dressings on early postoperative outcomes for the arthroscopic anterior cruciate ligament reconstruction patient. J Orthop Sports Phys Ther 27: 403-406, 1998.
26. di Chio F, Cecere A, Troiano M, Mardighian A, Parisi S and Guglielmi G: Persistent Haglund's disease after conventional treatments: The innovative role of radiotherapy. BJR Case Rep 2: 20150272, 2016.

27. Appala RS, Rajasekhara RG, Vijayabhushanam M, Venkateswara RM and Anil B: Comparision of open versus endoscopic calcaneoplasty for haglunds deformity: A short term analysis. J Evol Med Dent Sci 4: 1950-1954, 2015.

28. Bostick GP, Jomha NM, Suchak AA and Beaupre LA: Factors associated with calf muscle endurance recovery 1 year after achilles tendon rupture repair. J Orthop Sports Phys Ther 40: 345-351, 2010.

29. Boffeli TJ and Peterson MC: The Keck and Kelly wedge calcaneal osteotomy for Haglund's deformity: A technique for reproducible results. J Foot Ankle Surg 51: 398-401, 2012.

30. Ahn JH, Ahn CY, Byun CH and Kim YC: Operative treatment of Haglund syndrome with central achilles tendon-splitting approach. J Foot Ankle Surg 54: 1053-1056, 2015.

31. Sella EJ, Caminear DS and McLarney EA: Haglund's syndrome. J Foot Ankle Surg 37: 110-114; discussion 173, 1998.

This work is licensed under a Creative Commons Attribution-NonCommercial-NoDerivatives 4.0 International (CC BY-NC-ND 4.0) License. 